\title{
Rho GTPase-Activating Protein 35 rs 1052667 Polymorphism and Osteosarcoma Risk and Prognosis
}

\author{
Jinmin Zhao, ${ }^{1,2}$ Hua Xu, ${ }^{2,3}$ Maolin He, ${ }^{2,4}$ Zhe Wang, ${ }^{2,4}$ and Yang $\mathrm{Wu}^{2,4}$ \\ ${ }^{1}$ Department of Orthopaedics Trauma and Hand Surgery, The First Affiliated Hospital of Guangxi Medical University, \\ Nanning, Guangxi Zhuang Autonomous Region 530021, China \\ ${ }^{2}$ Research Center for Regenerative Medicine, Guangxi Medical University, Nanning, \\ Guangxi Zhuang Autonomous Region 530021, China \\ ${ }^{3}$ Center for Education Evaluation \& Faculty Development, Guangxi Medical University, Nanning, \\ Guangxi Zhuang Autonomous Region 530021, China \\ ${ }^{4}$ Division of Spinal Surgery, The First Affiliated Hospital of Guangxi Medical University, Nanning, \\ Guangxi Zhuang Autonomous Region 530021, China
}

Correspondence should be addressed to Maolin He; gxspine@foxmail.com

Received 5 February 2014; Revised 30 May 2014; Accepted 30 June 2014; Published 20 July 2014

Academic Editor: Urszula Demkow

Copyright (c) 2014 Jinmin Zhao et al. This is an open access article distributed under the Creative Commons Attribution License, which permits unrestricted use, distribution, and reproduction in any medium, provided the original work is properly cited.

The Rho GTPase-activating protein 35 (ARHGAP35), an important Rho family GTPase-activating protein, may be associated with tumorigenesis of some tumors. Here, we investigated the relationship between an important polymorphic variant at $3^{\prime}$-UTR of this gene (rs1052667) and osteosarcoma risk and prognosis. This hospital-based case-control study, including 247 osteosarcoma patients and 428 age-, sex-, and race-matched healthy controls, was conducted in Guangxi population. Genotypes were tested using TaqMan PCR technique. We found a significant difference in the frequency of rs1052667 genotypes between cases and controls. Compared with the homozygote of rs1052667 C alleles (rs1052667-CC), the genotypes with rs1052667 T alleles (namely, rs1052667-CT or -TT) increased osteosarcoma risk (odds ratios: 2.41 and 7.35, resp.). Moreover, rs1052667 polymorphism was correlated with such pathological features of osteosarcoma as tumor size, tumor grade, and tumor metastasis. Additionally, this polymorphism also modified the overall survival and recurrence-free survival of osteosarcoma cases. Like tumor grade, ARHGAP35 rs1052667 polymorphism was an independent prognostic factor influencing the survival of osteosarcoma. These results suggest that ARHGAP35 rs1052667 polymorphism may be associated with osteosarcoma risk and prognosis.

\section{Instruction}

Osteosarcoma is the most frequent primary malignant bone tumor and usually occurs in patients between 10 and 25 years of age $[1,2]$. In the past several years, the 5 -year survival of patients with osteosarcoma has significantly improved because of the combined treatment (neoadjuvant chemotherapy, surgery, and adjuvant chemotherapy) $[2,3]$. However, about $80 \%$ of patients would eventually develop metastatic disease following surgical treatment, and outcome remains poor for these patients [2-4].

Therefore, a better understanding of its basic biology is urgently needed to identify its risk and prognostic markers.
Several studies have reported potential associations of common genetic variants with osteosarcoma risk in biologically plausible pathways. This suggests that the genetic factors could play important roles in the pathogenesis of this malignant tumor $[5,6]$.

The Rho GTPase-activating protein 35 (ARHGAP35, also called GRLF1 and p190RhoGAP) is an important Rho family GTPase-activating protein, and is identified as a tyrosinephosphorylated protein associated with p120RasGAP in vSrc transformed cells [7-14]. Functionally, it mainly plays a crucial role in regulating cytoskeletal rearrangements, cell spreading and migration, and endothelial barrier function [15-18]. Recent data have shown that this protein can regulate 
cell proliferation and the dysregulation of ARHGAP35 may be associated with gliomas and breast cancer [19-21]. A common polymorphism at $3^{\prime}$-untranslated region $\left(3^{\prime}\right.$-UTR) of this gene, namely, rs1052667 $\mathrm{C}>\mathrm{T}$, has been identified. However, it is unclear whether this polymorphism correlates with tumor. Therefore, we specifically conducted a hospitalbased case-control study to examine whether ARHGAP35 rs1052667 polymorphism modifies osteosarcoma risk and prognosis.

\section{Materials and Methods}

2.1. Study Population. The present study was approved by the ethics committees of the hospitals involved in this study. This hospital-based case-control study was conducted in Guangxi Zhuang Autonomous Region, China, a relatively high incident area of osteosarcoma. All osteosarcoma patients and control individuals were residents of Guangxi Zhuang Autonomous Region and were recruited from the affiliated hospitals of Guangxi Medical University between January 1996 and August 2005. All cases were histopathologically confirmed. During the same period, control subjects without a history of cancer were randomly selected from a pool of healthy volunteers who visited the general health checkup center of the same hospitals because of their routine scheduled physical exams $[22,23]$. To control the effects of confounders, the controls were individually matched $(1: 1$ or $2: 1$ ) to cases based on ethnicity (Han, Minority), sex, and age ( \pm 5 years). In this study, a total of 247 cases and 428 controls, representing $97 \%$ of eligible cases and $92 \%$ of eligible controls, were enrolled, interviewed, and included in the final analysis. After giving written consent, demographic information and clinical pathological data (including age, sex, race, smoking and drinking status, disease history, tumor history, tumor size, tumor grade, and tumor site, etc.) were collected using a standard interviewer administered questionnaire and/or medical records. At the same time, $2 \mathrm{~mL}$ of peripheral blood was obtained for analyzing the genotypes of ARHGAP35 rs1052667 polymorphism. All subjects did not have chemotherapy or radiotherapy history before samples collection.

Among 247 osteosarcoma cases, about 57 percent (140/ 247) of osteosarcomas located in femur. All cases received surgical resection of primary tumor; however, only fiftyseven cases underwent the radical treatment (including both curative resection and adjuvant chemotherapy). In this study, tumor grade was evaluated according to Broders' grading system [24]. Low grade was defined as tumor type with well or moderately differentiated tumor cells (less than $50 \%$ undifferentiated cells), whereas high grade was defined as tumor type with poorly or anaplastic/pleomorphic differentiated tumor cells (more than 50\% undifferentiated cells) [25].

2.2. DNA Detraction. Leukocytes were isolated from peripheral venous blood samples from all tumor patients and control subjects by standard procedures. DNA was then extracted from leukocyte samples by standard phenol-chloroform extraction and ethanol precipitation. DNA samples were stored at $-20^{\circ} \mathrm{C}$ until additional analysis.

2.3. Genotyping. The ARHGAP35 rs1052667 genotypes were analyzed by TaqMan polymerase chain reaction (PCR) on an iCycler iQ real-time PCR detection system (iQ5, BioRad, Hercules, CA, USA). The corresponding TaqMan SNP Genotyping Assay Kit (cat\# 4351379) was obtained from Applied Biosystems, Carlsbad, CA, USA. TaqMan PCR was performed in total volume of $25 \mu \mathrm{L}$ consisting of $1 \times$ TaqMAN Universal Master Mix II (cat\# 4440041, Applied Biosystems), $1 \times$ TaqMan SNP Genotyping Assay Mix (including both primers and probes, cat\# C_16007053_10), and about $75 \mathrm{ng}$ of genomic DNA. Cycling conditions were $95^{\circ} \mathrm{C}$ for $30 \mathrm{~s}$, and 50 cycles of $95^{\circ} \mathrm{C}$ for $15 \mathrm{~s}$, and $60^{\circ} \mathrm{C}$ for $1 \mathrm{~min}$. For quality control, laboratory personnel were blinded to case and control status. Controls were included in each run, and repeated genotyping and sequencing of a random $20 \%$ subset yielded $100 \%$ identical genotypes.

2.4. Osteosarcoma Patients Follow-Up. For survival analysis, we followed all osteosarcoma cases. All patients underwent serial monitoring every 2 months for the first 2 years and semiannually thereafter for detection of any recurrence. In this study, the last follow-up day was December 31, 2013, and survival status was confirmed by clinic records and either patient or family contact. The duration of overall survival (OS) was defined as from the date of curative treatment to the date of death or last known date alive, whereas the recurrence-free survival (RFS) was defined as from the date of curative treatment to the date of tumor recurrence or last known date alive.

2.5. Statistical Analysis. All analyses were performed with the statistical package for social science (SPSS) version 18 (SPSS Institute, Chicago, IL, USA). Pearson's $\chi^{2}$ test or Fisher's exact test was used to test the differences between osteosarcoma patients and control subjects in the distribution of gender, age, race, and ARHGAP35 rs1052667 genotypes. Because this study was based on an individually matched design, conditional logistic regression was used to evaluate odds ratios (ORs) and 95\% confidence intervals (CIs) for risk of osteosarcoma. Kaplan-Meier survival analysis (with the log-rank test) was used to elucidate the relationship between ARHGAP35 rs1052667 polymorphism and osteosarcoma prognosis. Hazard ratios (HRs) and 95\% CIs for ARHGAP35 genotypes were calculated from a multivariate Cox regression model (with stepwise forward selection based on the likelihood ratio test). In the present study, a $P$ value of $<0.05$ was considered statistically significant.

\section{Results}

3.1. Demographic and Clinic Characteristics of the Subjects. In this study, 247 osteosarcoma cases and 428 controls were included in the final analysis. The demographic characteristics of all cases and controls are shown in Table 1. The mean age, gender ratio, smoking and drinking status, and 
TABLE 1: Demographic and etiologic characteristics of osteosarcoma cases and controls.

\begin{tabular}{|c|c|c|c|c|c|}
\hline \multirow{2}{*}{ Variable } & \multicolumn{2}{|c|}{ Controls $(n=428)$} & \multicolumn{2}{|c|}{ Cases $(n=247)$} & \multirow{2}{*}{$P$} \\
\hline & $n$ & $\%$ & $n$ & $\%$ & \\
\hline \multicolumn{6}{|l|}{ Sex } \\
\hline Male & 265 & 61.9 & 154 & 62.3 & \multirow{2}{*}{0.934} \\
\hline Female & 163 & 38.1 & 93 & 37.7 & \\
\hline \multicolumn{6}{|l|}{ Age (yrs) } \\
\hline$\leq 26$ & 278 & 65.0 & 161 & 65.2 & \multirow{2}{*}{0.952} \\
\hline$>26$ & 150 & 35.0 & 86 & 34.8 & \\
\hline \multicolumn{6}{|l|}{ Race } \\
\hline Han & 279 & 65.2 & 159 & 64.4 & \multirow{2}{*}{0.555} \\
\hline Minority & 149 & 34.8 & 88 & 35.6 & \\
\hline \multicolumn{6}{|c|}{ Smoking status } \\
\hline No & 398 & 93.0 & 231 & 93.5 & \multirow{2}{*}{0.792} \\
\hline Yes & 30 & 7.0 & 16 & 6.5 & \\
\hline \multicolumn{6}{|c|}{ Drinking status } \\
\hline No & 402 & 93.9 & 233 & 94.3 & \multirow{2}{*}{0.829} \\
\hline Yes & 26 & 6.1 & 14 & 5.7 & \\
\hline \multicolumn{6}{|c|}{ Paget's disease } \\
\hline No & 428 & 100.0 & 246 & 96.6 & \multirow{2}{*}{0.366} \\
\hline Yes & 0 & 0.0 & 1 & 0.4 & \\
\hline \multicolumn{6}{|l|}{ Trauma } \\
\hline No & 411 & 96.0 & 228 & 92.3 & \multirow{2}{*}{0.038} \\
\hline Yes & 17 & 4.0 & 19 & 7.7 & \\
\hline \multicolumn{6}{|c|}{ Radiation exposure } \\
\hline No & 415 & 97.0 & 236 & 95.5 & \multirow{2}{*}{0.339} \\
\hline Yes & 13 & 3.0 & 11 & 4.5 & \\
\hline \multicolumn{6}{|l|}{$\mathrm{PBBL}^{\mathrm{b}}$} \\
\hline No & 428 & 100.0 & 246 & 96.6 & \multirow{2}{*}{0.366} \\
\hline Yes & 0 & 0.0 & 1 & 0.4 & \\
\hline
\end{tabular}

${ }^{\mathrm{a}}$ The mean \pm S.D. ages were $26.38 \pm 15.51$ and $26.31 \pm 14.16$ for cases and controls, respectively.

${ }^{\mathrm{b}} \mathrm{PBBL}$ refers to the preexisting benign bone lesions, including fibrous dysplasia, osteochondromatosis, and chondromatosis.

TABLE 2: The rs1052667 polymorphism of ARHGAP35 and osteosarcoma risk.

\begin{tabular}{|c|c|c|c|c|c|c|}
\hline \multirow{2}{*}{ rs1052667 } & \multicolumn{2}{|c|}{ Controls } & \multicolumn{2}{|c|}{ Cases } & \multirow{2}{*}{ OR } & \multirow{2}{*}{$P$} \\
\hline & $n$ & $\%$ & $n$ & $\%$ & & \\
\hline \multicolumn{7}{|l|}{ Genotype } \\
\hline CC & 341 & 79.7 & 133 & 53.8 & 1 & \\
\hline $\mathrm{CT}$ & 72 & 16.8 & 70 & 28.3 & $2.41(1.64-3.55)^{\mathrm{a}}$ & $9.00 \times 10^{-6}$ \\
\hline $\mathrm{TT}$ & 15 & 3.5 & 44 & 17.8 & $7.35(3.95-13.68)^{\mathrm{a}}$ & $3.12 \times 10^{-10}$ \\
\hline $\mathrm{CT} / \mathrm{TT}^{\mathrm{b}}$ & 87 & 20.3 & 114 & 46.2 & $3.27(2.31-4.61)^{\mathrm{a}}$ & $1.92 \times 10^{-11}$ \\
\hline \multicolumn{7}{|l|}{ Allele } \\
\hline C & 754 & 88.1 & 336 & 68.0 & 1 & \\
\hline $\mathrm{T}$ & 102 & 11.9 & 158 & 32.0 & $2.25(1.64-3.09)$ & $4.43 \times 10^{-7}$ \\
\hline
\end{tabular}

${ }^{a}$ OR conditional on matched set adjusted by smoking and drinking status, radiation exposure history, trauma history, paget's disease history, and benign bone lesions.

${ }^{\mathrm{b}} \mathrm{CT} / \mathrm{TT}$ represented the combination of rs1052667-CT genotype and rs1052667-TT genotype.

race distribution are of the same levels in both control and osteosarcoma groups $(P>0.05)$.

3.2. ARHGAP35 Polymorphism Increased Osteosarcoma Risk. Table 2 summarized the genotypic and allelic distribution of ARHGAP35 rs1052667 polymorphism for both tumor patients and controls. Genotype frequent distribution in controls fitted the Hardy-Weinberg equilibrium well. The heterozygous genotype with rs1052667 C and T allele (rs1052667CT) and the variant homozygous genotype with rs1052667 
TABLE 3: The rs1052667 polymorphism of ARHGAP35 and osteosarcoma risk stratified by race (Han and minority), gender (female and male), and age ( $\leq 26$ yrs and $>26$ yrs).

\begin{tabular}{|c|c|c|c|c|c|c|c|}
\hline \multirow{2}{*}{ Variable } & \multirow{2}{*}{ Genotype } & \multicolumn{2}{|c|}{ Control } & \multicolumn{2}{|c|}{ Case } & \multirow{2}{*}{ OR $(95 \% \mathrm{CI})^{\mathrm{a}}$} & \multirow{2}{*}{$P$} \\
\hline & & $n$ & $\%$ & $n$ & $\%$ & & \\
\hline Race $^{b}$ & rs1052667 & & & & & & \\
\hline \multirow{2}{*}{ Han } & CC & 218 & 78.1 & 82 & 51.6 & 1 & \\
\hline & $\mathrm{CT} / \mathrm{TT}$ & 61 & 21.9 & 77 & 48.4 & $3.40(2.22-5.19)$ & $1.61 \times 10^{-8}$ \\
\hline \multirow{2}{*}{ Minority } & CC & 123 & 82.6 & 51 & 58.0 & 1 & \\
\hline & $\mathrm{CT} / \mathrm{TT}$ & 26 & 17.4 & 37 & 42.0 & $3.39(1.86-6.18)$ & $6.67 \times 10^{-5}$ \\
\hline Gender $^{c}$ & rs1052667 & & & & & & \\
\hline \multirow{2}{*}{ Female } & CC & 129 & 79.1 & 49 & 52.7 & 1 & \\
\hline & $\mathrm{CT} / \mathrm{TT}$ & 34 & 20.9 & 44 & 47.3 & $3.42(1.96-5.98)$ & $1.53 \times 10^{-5}$ \\
\hline \multirow{2}{*}{ Male } & CC & 212 & 80.0 & 84 & 54.5 & 1 & \\
\hline & $\mathrm{CT} / \mathrm{TT}$ & 53 & 20.0 & 70 & 45.5 & $3.29(2.12-5.12)$ & $1.08 \times 10^{-7}$ \\
\hline $\operatorname{Age}^{\mathrm{d}}$ & rs1052667 & & & & & & \\
\hline \multirow{2}{*}{$\leq 26$} & CC & 214 & 77.0 & 80 & 49.7 & 1 & \\
\hline & $\mathrm{CT} / \mathrm{TT}$ & 64 & 23.0 & 81 & 50.3 & $3.40(2.23-5.17)$ & $1.11 \times 10^{-8}$ \\
\hline \multirow{2}{*}{$>26$} & CC & 127 & 84.7 & 53 & 61.6 & 1 & \\
\hline & $\mathrm{CT} / \mathrm{TT}$ & 23 & 15.3 & 33 & 38.4 & $3.35(1.79-6.25)$ & $1.50 \times 10^{-4}$ \\
\hline $\begin{array}{l}{ }^{\mathrm{a}} \text { OR condition } \\
{ }^{\mathrm{b}} \text { Likelihood rat } \\
\text { across strata (ir } \\
{ }^{\mathrm{c}} \text { Likelihood rat } \\
\text { across strata (ir } \\
{ }^{\mathrm{d}} \text { Likelihood rat } \\
\text { ORs across stra }\end{array}$ & $\begin{array}{l}\text { ched set. } \\
\mathrm{r} \text { interaction } \\
\mathrm{rm} \mathrm{OR}=1.02 \\
\mathrm{r} \text { interaction } \\
\mathrm{rm} \mathrm{OR}=0.99 \\
\mathrm{r} \text { interaction } \\
\mathrm{ct} \text { term } \mathrm{OR}=\end{array}$ & $\begin{array}{l}=0 . \\
\text { tified } \\
=0 \\
\text { ified }\end{array}$ & $\begin{array}{l}\text { Ian an } \\
\text { nale a } \\
\text { ge: } \leq 2\end{array}$ & $\begin{array}{l}\text { and } \\
26 y 1\end{array}$ & $\begin{array}{l}\text { geno } \\
\text { genot } \\
52667\end{array}$ & $\begin{array}{l}\text { Iculated as test for } \\
\text { Iculated as test for } \\
\text { vas calculated as tes }\end{array}$ & $\begin{array}{l}\text { geneity of ORs } \\
\text { geneity of ORs } \\
\text { eterogeneity of }\end{array}$ \\
\hline
\end{tabular}

T allele (rs1052667-TT) were more frequent among cases than among the controls $(P<0.01)$, resulting in an Ser allele frequency of $32.0 \%$ in cases and $11.9 \%$ in controls. Logistic regression analysis exhibited that the adjusted OR for osteosarcoma for these individuals carrying rs1052667CT compared with those exhibiting the homozygote for C alleles (rs1052667-CC) was 2.41 (95\% CI, 1.64-3.55) and the corresponding OR for those featuring rs1052667-TT was 7.35 (95\% CI, 3.95-13.68). These results showed that osteosarcoma risk was associated with the number of rs1052667 T alleles.

3.3. ARHGAP35 Polymorphism and Osteosarcoma Risk Stratified by Gender, Age, and Race. To evaluate possible interactive effects of matching factors (including gender, age, and race) and ARHGAP35 rs1052667 polymorphism on osteosarcoma risk, we performed a series of bivariate stratified analyses by matching factors (Table 3 ). Because of the small number of subjects with rs1052667-TT among different strata, genotypes rs1052667-CT and rs1052667-TT were combined into one stratum (also called rs1052667-CT/TT). Similar risk values for osteosarcoma were found among Han subjects and among minority participants (adjusted ORs were 3.40 and 3.39 , resp.). Similar results were also found in the stratified analysis between rs1052667 polymorphism and other two matching variables. Likelihood ratio tests for interaction of the stratified variables and ARHGAP35 genotypes showed that these matching factors did not modulate the effects of this polymorphism on osteosarcoma risk $\left(P_{\text {interaction }}>0.05\right.$;
Table 3). This suggested that these factors should be effectually manipulated and should not modify the association between this polymorphism and osteosarcoma risk.

3.4. ARHGAP35 Polymorphism Modified Osteosarcoma Prognosis. To investigate the effects of ARHGAP35 polymorphism on outcome of osteosarcoma patients, we followed all cases and analyzed the survival information of all osteosarcoma cases. During the follow-up period of these patients, 222 faced tumor recurrence with $15.9 \%$ of the 5year RFS rate, and 238 died with $12.5 \%$ of the five-year OS rate. Kaplan-Meier survival analysis showed that patients with ARHGAP35 rs1052667 T alleles featured a significantly poorer prognosis than those with rs1052667-CC $(P$ is $1.19 \times$ $10^{-11}$ for OS and $P$ is $2.04 \times 10^{-17}$ for RFS, resp.; Figures 1(a) and 1(b)). Considering that some patients did not accomplish entire adjuvant chemotherapy because of poor economic conditions, we stratified the analysis of the correlation between ARHGAP35 genotypes and osteosarcoma outcome by the radical treatment status to explore whether this difference affected the results (Figure 2). Among these cases receiving the radical treatment (Figures 2(c) and 2(d)), shorter median overall survival time (MST) and shorter median tumor recurrence-free survival time (MRT) were found in cases having risk genotypes (including ARHGAP35 rs1052667-CT and -TT) than in those without risk genotypes. Similar results were observed in the nonradical treatment 


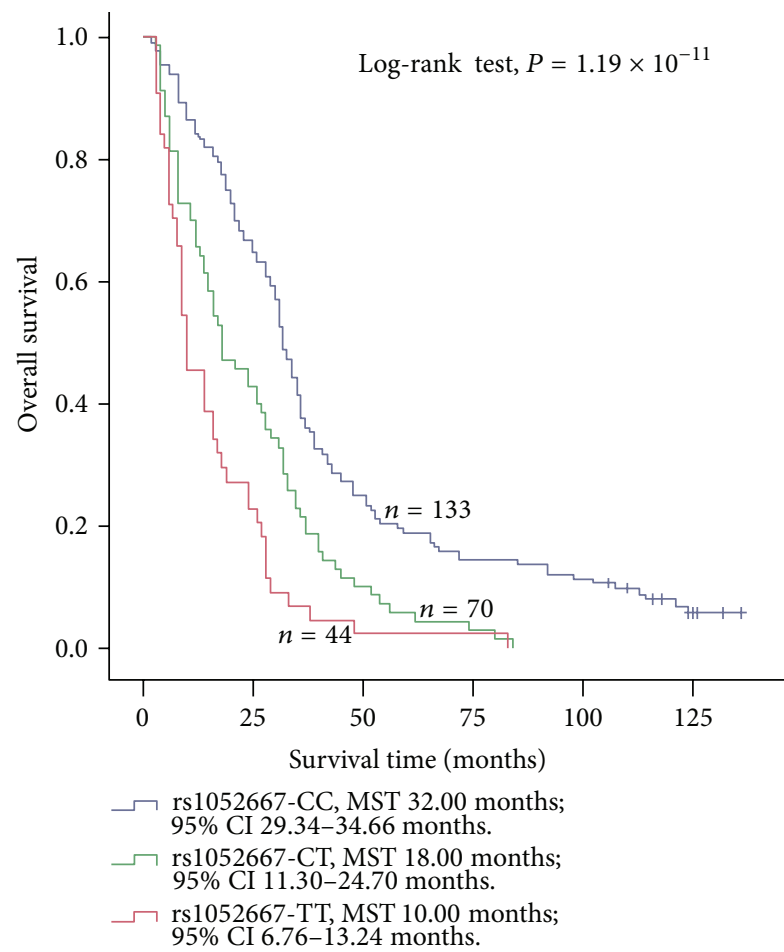

(a)

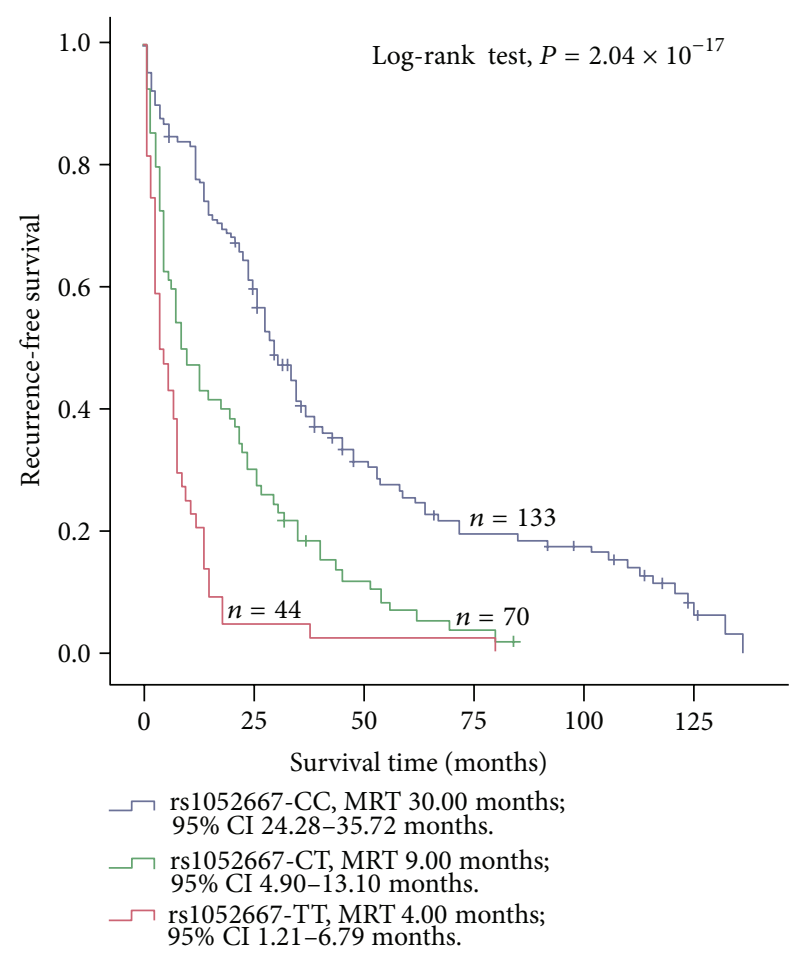

(b)

FIGURE 1: Association between ARHGAP35 rs1052667 polymorphism and osteosarcoma prognosis in 247 osteosarcoma patients. ARHGAP35 rs1052667 polymorphism was correlated with (a) the overall survival and (b) the recurrence-free survival of osteosarcoma. Cumulative hazard function was plotted by the Kaplan-Meier methodology and the $P$ value was calculated with two-sided log-rank tests. MST, the median overall survival time; MRT, the median tumor recurrence-free survival time.

TABLE 4: The rs1052667 polymorphism of ARHGAP35 and the prognosis of osteosarcoma.

\begin{tabular}{lcccc}
\hline Rs1052667 & \multicolumn{2}{c}{ Overall survival } & \multicolumn{2}{c}{ Recurrence-free survival } \\
Genotype & HR $(95 \%$ CI $)$ & $P$ & HR $(95 \%$ CI $)$ & $P$ \\
\hline CC & 1 & & 1 & \\
CT & $1.57(1.16-2.12)$ & $3.86 \times 10^{-3}$ & $1.82(1.33-2.50)$ & $2.12 \times 10^{-4}$ \\
TT & $1.91(1.32-2.77)$ & $5.95 \times 10^{-4}$ & $2.53(1.73-3.70)$ & $1.73 \times 10^{-6}$ \\
\hline
\end{tabular}

stratum (Figures 2(a), and 2(b)). Multivariate cox regression analysis (with stepwise forward selection based on likelihood ratio test) was next performed to determine whether ARHGAP35 rs1052667 polymorphism was an independent predictor of osteosarcoma cases. The results exhibited that the genotypes with rs1052667 $\mathrm{T}$ alleles increased the dying risk of tumor patients compared with rs1052667-CC (HRs: 1.57 for rs1052667-CT and 1.91 for rs1052667-TT, resp.). Risk role was also found in the RFS analysis; the corresponding HRs were 1.82 for rs1052667-CT and 2.53 for rs1052667-TT, respectively (Table 4). Taken together, these results implied that this polymorphism could be used as an independent prognostic marker for osteosarcoma.

3.5. ARHGAP35 Polymorphism Correlated with the ClinicPathological Features of Osteosarcoma Patients. To explore whether ARHGAP35 rs1052667 polymorphism correlated with the clinical pathological features of osteosarcoma, an association analysis of the risk genotypes (rs1052667-CT/TT) or the nonrisk genotype (rs1052667-CC) and the clinical pathological characteristics of osteosarcoma was performed separately. Results showed that these osteosarcoma cases with risk genotypes of ARHGAP35, compared to those without risk genotypes, faced larger tumor size (OR is 4.85), lower tumor differentiation (OR is 4.07), and higher metastasis risk (OR is 2.78; Table 5). However, this polymorphism did not affect other features.

\section{Discussion}

To the best of our knowledge, no studies have investigated the role of ARHGAP35 rs1052667 polymorphism in the risk of osteosarcoma. In this study, we analyzed the association between aforementioned polymorphism and the risk of osteosarcoma among Guangxi population and found ARHGAP35 rs1052667 T alleles increased osteosarcoma risk (adjusted OR is 3.27). These results imply that this polymorphism may have functional significance in osteosarcoma carcinogenesis.

Osteosarcoma is one of major cancer types in the Guangxi Zhuang Autonomous Region; the possible risk factors of which include radiation exposure, foreign bodies, genetic predisposition, and so on. Increasing epidemiological evidence has shown that an individual susceptibility related 


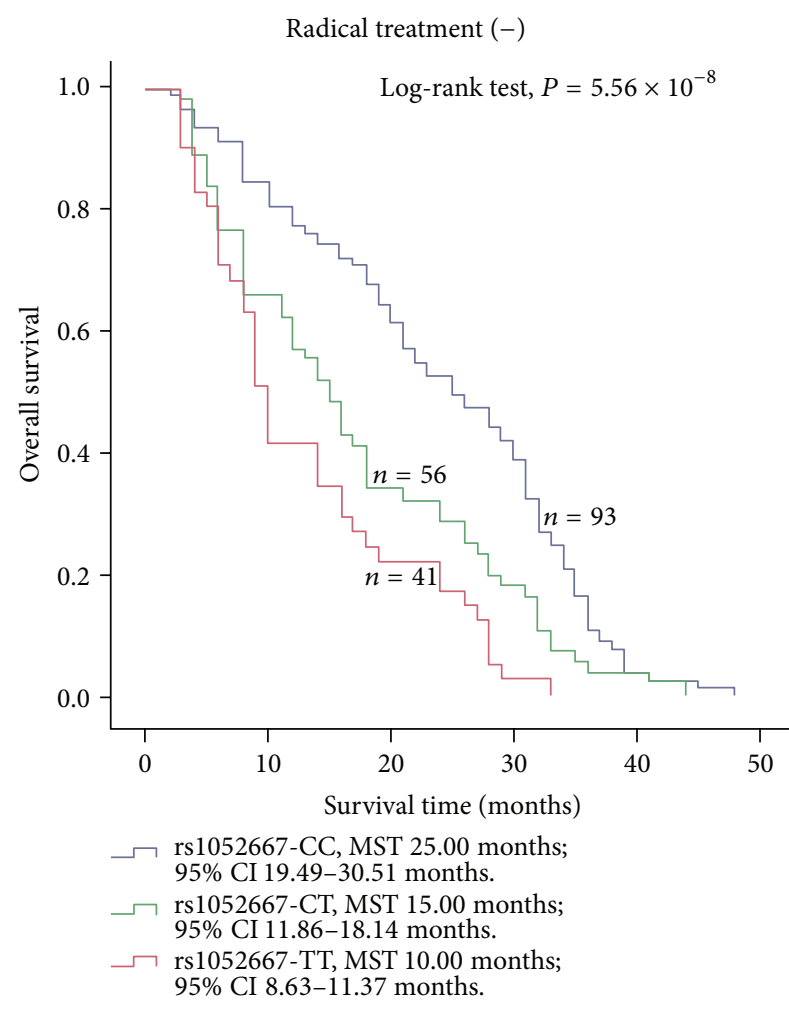

(a)

Radical treatment $(+)$

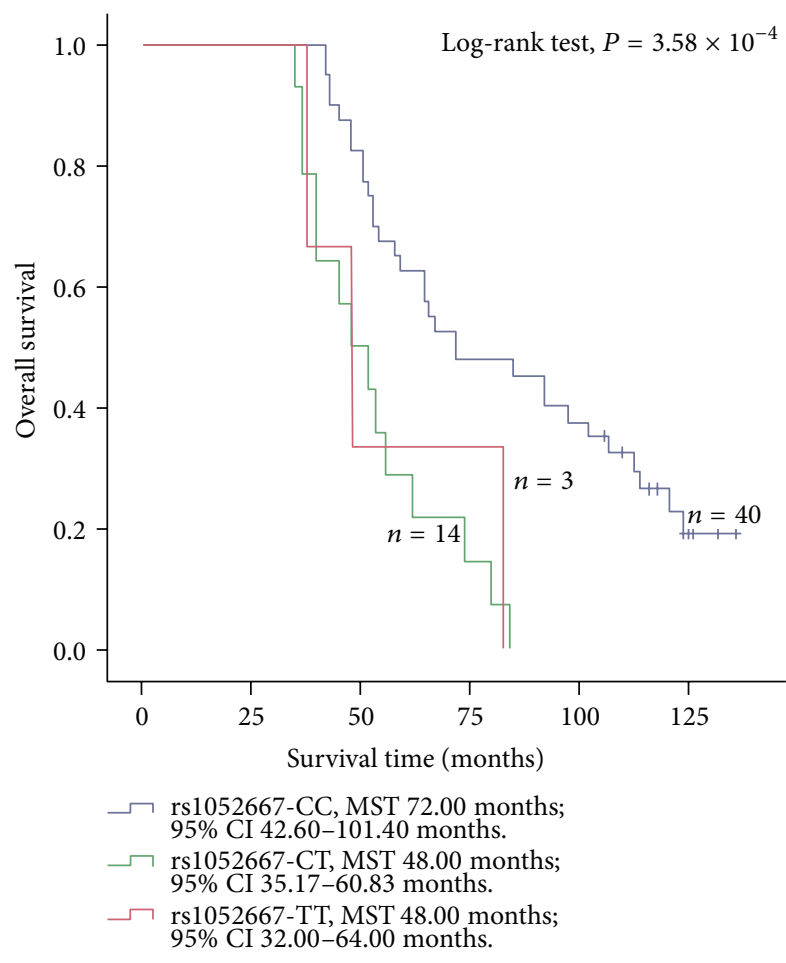

(c)

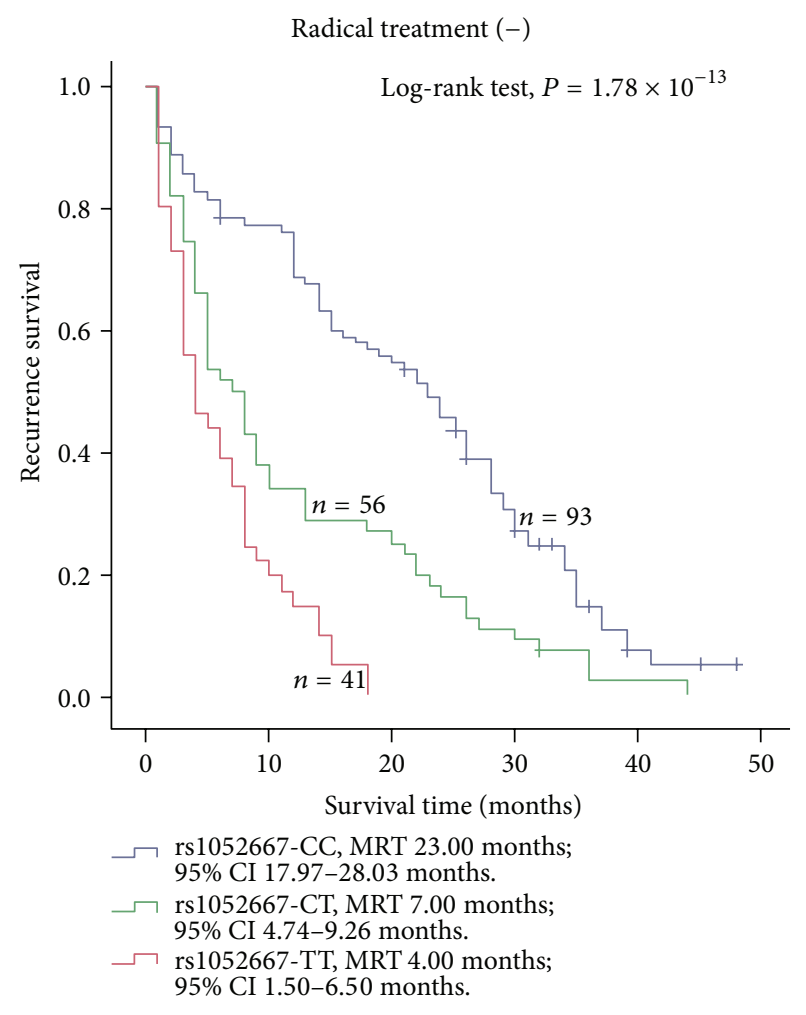

(b)

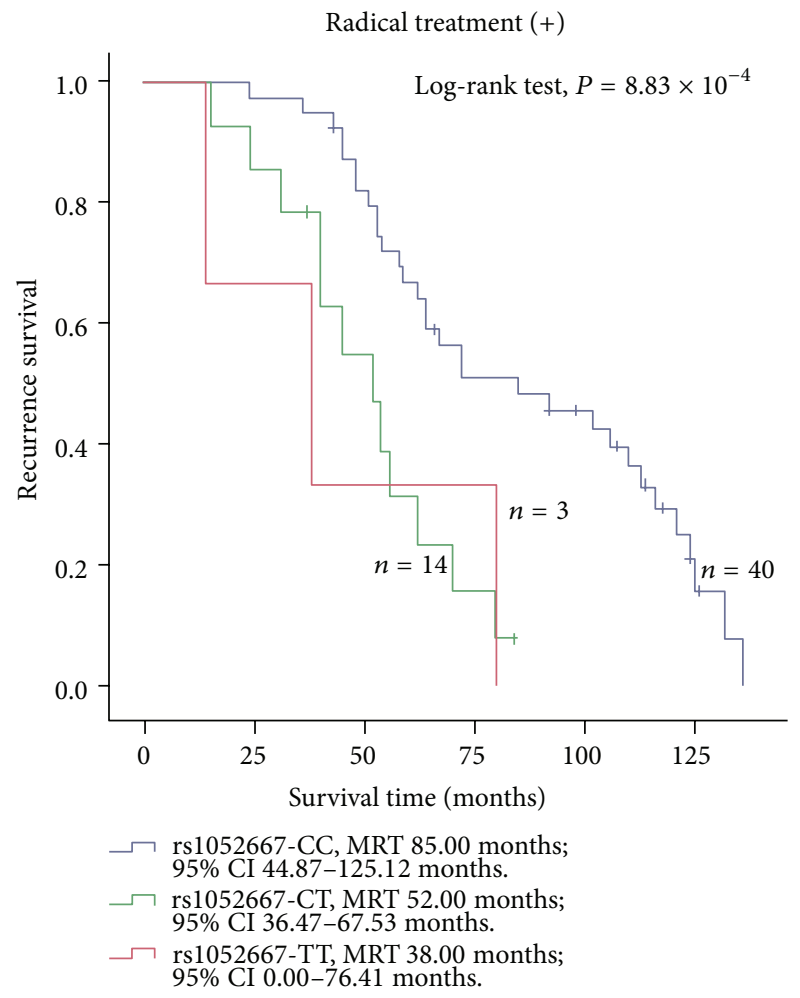

(d)

FIGURE 2: Survival analysis of ARHGAP35 rs1052667 polymorphism in strata of treatment status. According to whether cases received radical treatment (RT) including both surgical resection and adjuvant chemotherapy, 247 osteosarcoma cases were divided into two groups: RT (+) and RT (-). ((a), (c)) Overall survival and ARHGAP35 rs1052667 polymorphism in strata of RT status. ((b), (d)) Tumor recurrencefree survival and ARHGAP35 rs1052667 polymorphism in strata of status. Cumulative hazard function was plotted by Kaplan-Meier's methodology, and $P$ value was calculated with two-sided log-rank tests. MST, the median overall survival time; MRT, the median tumor recurrence-free survival time. 
TABLE 5: The rs1052667 polymorphism of ARHGAP35 and clinic pathological features of osteosarcoma.

\begin{tabular}{|c|c|c|c|c|c|c|}
\hline \multirow{2}{*}{ Variable } & \multicolumn{2}{|c|}{ rs1052667-CC } & \multicolumn{2}{|c|}{ rs1052667-CT/TT } & \multirow{2}{*}{ OS (95\% CI) } & \multirow{2}{*}{$P$} \\
\hline & $n$ & $\%$ & $n$ & $\%$ & & \\
\hline \multicolumn{7}{|l|}{ Age (yrs) } \\
\hline$\leq 26$ & 80 & 60.2 & 81 & 71.1 & 1 & \\
\hline$>26$ & 53 & 39.8 & 33 & 28.9 & $0.72(0.39-1.35)$ & 0.31 \\
\hline \multicolumn{7}{|l|}{ Gender } \\
\hline Female & 49 & 36.8 & 44 & 38.6 & 1 & \\
\hline Male & 84 & 63.2 & 70 & 61.4 & $1.07(0.61-1.89)$ & 0.82 \\
\hline \multicolumn{7}{|l|}{ Race } \\
\hline Han & 82 & 61.7 & 77 & 67.5 & 1 & \\
\hline Minority & 51 & 38.3 & 37 & 32.5 & $0.98(0.54-1.77)$ & 0.95 \\
\hline \multicolumn{7}{|l|}{ Tumor site } \\
\hline Femur & 72 & 54.1 & 68 & 59.6 & 1 & \\
\hline Tibia & 30 & 22.6 & 21 & 18.4 & $0.66(0.32-1.33)$ & 0.24 \\
\hline Humeral bone & 22 & 16.5 & 16 & 14 & $0.90(0.40-2.01)$ & 0.79 \\
\hline Others & 9 & 6.8 & 9 & 7.9 & $0.91(0.31-2.70)$ & 0.86 \\
\hline \multicolumn{7}{|l|}{ Tumor size } \\
\hline$\leq 5 \mathrm{~cm}$ & 57 & 42.9 & 15 & 13.2 & 1 & \\
\hline$>5 \mathrm{~cm}$ & 76 & 57.1 & 99 & 86.8 & $4.85(2.51-9.37)$ & $2.69 \times 10^{-6}$ \\
\hline \multicolumn{7}{|l|}{ Tumor grade } \\
\hline Low & 68 & 51.1 & 23 & 20.2 & 1 & \\
\hline High & 65 & 48.9 & 91 & 79.8 & $4.07(2.28-7.37)$ & $2.14 \times 10^{-6}$ \\
\hline \multicolumn{7}{|l|}{ Metastasis } \\
\hline No & 87 & 65.4 & 45 & 39.5 & 1 & \\
\hline Yes & 46 & 34.6 & 69 & 60.2 & $2.78(1.63-4.76)$ & $1.88 \times 10^{-4}$ \\
\hline
\end{tabular}

to genetic factors might be associated with osteosarcoma carcinogenesis $[5,6]$.

While ARHGAP35 spans $87 \mathrm{~kb}$ on chromosome 19q13.3 and contains 7 exons and 6 introns (PubMed Databases). Its encoding protein is a $190 \mathrm{kDa}$ protein consisting of three major functional domains: (1) an $\mathrm{NH}_{2}$-terminal GTP-binding domain (GBD), (2) a middle domain (MD), and (3) a $\mathrm{COOH}$-terminal GAP domain, which displays specificity for GTP-bound RhoA [9, 26]. Functionally, ARHGAP35 plays important roles in promoting cell spreading, membrane protrusion, and cell polarity $[15,27]$. Recently, several reports have shown that ARHGAP35 plays an important role in cancer formation and metastasis [19, 20, 28]. In 2008, Shen et al. [19] investigated the role of ARHGAP35 in the breast tumor kinase (Brk) signal pathway and found that it is a Brk substrate both in vitro and in vivo. Through this signal pathway, ARHGAP35 is phosphorylated at the Y1105 residue by Brk and next associated with p120RasGAP. As a consequence, ARHGAP35 is stimulated and p120 functions are attenuated, leading to RhoA inactivation and Ras activation, respectively. Their results show ARHGAP35 activation promotes breast cancer growth, migration, and invasion, and provide important evidence for the crucial roles of this Brk-ARHGAP35 signaling pathway in promoting breast malignancy [19]. In accordance with these reports, our present study exhibited that ARHGAP35 might be involved in osteosarcoma tumorigenesis.
With the Human Genome Project developing, more than one hundred polymorphisms have been identified in ARHGAP35 (dbSNP in NCBI Database). In this study, we only analyzed ARHGAP35 rs1052667 polymorphism, primarily because this polymorphism is relatively common in most populations, whereas other polymorphisms are rare. In this study, we collected 247 osteosarcoma and 428 control samples from Guangxi Zhuang Autonomous Region, a relatively high incident area of osteosarcoma. we found that about 20 percent of control individuals had ARHGAP35 rs1052667 $\mathrm{T}$ alleles, similar to the data from the Human Genome Project (dbSNP Database, web: http://www.ncbi .nlm.nih.gov/SNP/snp_ref.cgi?rs=rs1052667). However, higher frequency was observed in the individuals with osteosarcoma, and following analysis showed this polymorphism increased osteosarcoma risk. These results suggested ARHGAP35 rs1052667 polymorphism might modify the risk of tumors such as osteosarcoma.

This risk role might be related to the posttranscriptional regulation of gene expression. Because rs1052667 polymorphism locates at the $3^{\prime}$-UTR of ARHGAP35 gene, this variant might be involved in the regulation of mRNA stability and the control of mRNA subcellular localization [29]. Consequently, it may be associated with the functional dysregulation of ARHGAP35 and play a role in the carcinogenesis. Supporting the aforementioned hypothesis, recent studies have shown that the dysregulation of ARHGAP35 expression and function is involved in the tumorigenesis of some tumors 
such as lung cancer [30], melanoma [31], and breast cancer $[19,28,32]$. Thus, ARHGAP35 polymorphism might play an important role in the tumorigenesis of osteosarcoma, and this provided a new genetic insight into osteosarcoma tumorigenesis.

Additionally, we also investigated the association between ARHGAP35 rs1052667 polymorphism and osteosarcoma prognosis. We found that osteosarcoma patients having genotypes with ARHGAP35 rs1052667 T alleles had a significant poor RFS and OS compared to those without $\mathrm{T}$ alleles. Considering the difference of the treatment and to explore whether this difference affected the modifying role of ARHGAP35 rs1052667 polymorphism, we stratified the analysis of the effects of ARHGAP35 genotypes on osteosarcoma outcome by the treatment status. Results showed that this polymorphism modulated osteosarcoma prognosis, regardless of the radical or nonradical treatment status. Multivariate cox regression analysis next showed this polymorphism increased 1.53-times tumor reoccurrence risk and 0.91-times death risk; moreover, this risk did not depend on the clinical pathological change. This is possibly because it correlates with the fact that this polymorphism modifies tumor grade and differentiation and, consequently, might promote tumor proliferation and metastasis. Supporting our results, recent studies have exhibited that the dysregulation of ARHGAP35 promotes tumor growth, infiltration, and metastases and subsequently might result in poor prognosis of tumors [19, 20, 28, 30]. These data implied that ARHGAP35 rs1052667 polymorphism should be able to modify the prognosis of osteosarcoma and should be an important prognostic marker for this tumor.

In the present study, to control the effects of confounders such as age, gender, and race, we used an individually matched design. In the stratified analysis, no interactive effects were found, suggesting that these factors do not modify the correlation between ARHGAP35 rs1052667 polymorphism and osteosarcoma risk.

However, there were several limitations to our study. Potential selection bias might have occurred because the selection of control subjects in our study was hospital-based. Despite the analysis of ARHGAP35 rs1052667 polymorphism, we did not analyze other polymorphisms of this gene possibly able to modify the risk of osteosarcoma. Although this study is molecular epidemiological investigation based on clinic samples of osteosarcomas, it is deficient in functional analysis. Additionally, our findings were based on relatively small numbers and limited by small number of subjects in part of the genotype strata. Therefore, more genes deserve further elucidation based on a large sample and the combination of genes.

\section{Conclusions}

In summary, to the best of our knowledge, this is the first report investigating an association between ARHGAP35 rs1052667 polymorphism and osteosarcoma risk and prognosis in Guangxi patients. We have found evidence that the genotypes of ARHGAP35 rs1052667 T alleles may be correlated with increased risk and poor prognosis for osteosarcoma and that this polymorphism may be involved in the tumorigenesis of this type of tumor. Given that osteosarcoma is a highly fatal tumor, the finding of a genetic susceptibility (if confirmed) may have implications for screening and prevention.

\section{Conflict of Interests}

The authors declare that there is no conflict of interests regarding the publication of this paper.

\section{Authors' Contribution}

Jinmin Zhao and Hua Xu contributed equally to this work.

\section{Acknowledgments}

The authors thank Yong-Chang Zhang for molecular biochemical technique. The authors also thank Xi-Dai Long for reading and comments of this paper. This work was supported in part by National Natural Science Foundation of China (no. 81160323).

\section{References}

[1] R. Gorlick, K. Janeway, S. Lessnick, R. L. Randall, and N. Marina, "Children's oncology group's 2013 blueprint for research: bone tumors," Pediatric Blood \& Cancer, vol. 60, no. 6, pp. 1009-1015, 2013.

[2] D. S. Geller and R. Gorlick, "Osteosarcoma: a review of diagnosis, management, and treatment strategies," Clinical Advances in Hematology and Oncology, vol. 8, no. 10, pp. 705-718, 2010.

[3] Y. Cho, G. Jung, S. Chung, J. Kim, and Y. Choi, "Long-term survivals of stage IIb osteosarcoma: a 20-year experience in a single institution," Clinics in Orthopedic Surgery, vol. 3, no. 1, pp. 48-54, 2011.

[4] F. Lamoureux, V. Trichet, C. Chipoy, F. Blanchard, F. Gouin, and F. Redini, "Recent advances in the management of osteosarcoma and forthcoming therapeutic strategies," Expert Review of Anticancer Therapy, vol. 7, no. 2, pp. 169-181, 2007.

[5] Z. Chang, R. Song, S. Xu, M. Xu, and X. Yu, "CD 152 gene polymorphisms and risk of osteosarcoma in Chinese population," Tumor Biology, 2014.

[6] Z. Wang, P. Wen, X. Luo et al., "Association of the vascular endothelial growth factor (VEGF) gene single-nucleotide polymorphisms with osteosarcoma susceptibility in a Chinese population," Tumour Biology, vol. 35, no. 4, pp. 3605-3610, 2014.

[7] T. C. Hou, J. J. Lin, H. C. Wen, L. C. Chen, S. P. Hsu, and W. S. Lee, "Folic acid inhibits endothelial cell migration through inhibiting the RhoA activity mediated by activating the folic acid receptor/cSrc/p190RhoGAP- signaling pathway," Biochemical Pharmacology, vol. 85, no. 3, pp. 376-384, 2013.

[8] I. Oinuma, K. Kawada, K. Tsukagoshi, and M. Negishi, "Rnd1 and Rnd3 targeting to lipid raft is required for p190 RhoGAP activation," Molecular Biology of the Cell, vol. 23, no. 8, pp. 15931604, 2012. 
[9] K. L. Grinnell and E. O. Harrington, "Interplay between FAK, PKC 8 , and p190RhoGAP in the regulation of endothelial barrier function," Microvascular Research, vol. 83, no. 1, pp. 12-21, 2012.

[10] J. Selva and G. Egea, "Ethanol increases p190RhoGAP activity, leading to actin cytoskeleton rearrangements," Journal of Neurochemistry, vol. 119, no. 6, pp. 1306-1316, 2011.

[11] T. Németh, K. Futosi, C. Hably et al., "Neutrophil functions and autoimmune arthritis in the absence of p190RhoGAP: Generation and analysis of a novel null mutation in mice," Journal of Immunology, vol. 185, no. 5, pp. 3064-3075, 2010.

[12] H. Naoe, K. Araki, O. Nagano et al., "The anaphase-promoting complex/cyclosome activator Cdh1 modulates Rho GTPase by targeting p190 RhoGAP for degradation," Molecular and Cellular Biology, vol. 30, no. 16, pp. 3994-4005, 2010.

[13] S. A. S. Manchinelly, J. A. Miller, L. Su et al., "Mitotic downregulation of p190RhoGAP is required for the successful completion of cytokinesis," Journal of Biological Chemistry, vol. 285, no. 35, pp. 26923-26932, 2010.

[14] A. Tomar, S. Lim, Y. Lim, and D. D. Schlaepfer, "A FAKp120RasGAP-p190RhoGAP complex regulates polarity in migrating cells," Journal of Cell Science, vol. 122, part 11, pp. 1852-1862, 2009.

[15] W. T. Arthur and K. Burridge, "RhoA inactivation by p190RhoGAP regulates cell spreading and migration by promoting membrane protrusion and polarity," Molecular Biology of the Cell, vol. 12, no. 9, pp. 2711-2720, 2001.

[16] G. A. Wildenberg, M. R. Dohn, R. H. Carnahan et al., "p120Catenin and p190RhoGAP Regulate Cell-Cell Adhesion by Coordinating Antagonism between Rac and Rho," Cell, vol. 127, no. 5, pp. 1027-1039, 2006.

[17] A. Mammoto, S. Huang, and D. E. Ingber, "Filamin links cell shape and cytoskeletal structure to Rho regulation by controlling accumulation of p190RhoGAP in lipid rafts," Journal of Cell Science, vol. 120, no. 3, pp. 456-467, 2007.

[18] A. K. Fordjour and E. O. Harrington, "PKC $\delta$ influences p190 phosphorylation and activity: events independent of PKC $\delta$ mediated regulation of endothelial cell stress fiber and focal adhesion formation and barrier function," Biochimica et Biophysica Acta-General Subjects, vol. 1790, no. 10, pp. 1179-1190, 2009.

[19] C. Shen, H. Chen, M. Lin et al., "Breast tumor kinase phosphorylates p190RhoGAP to regulate Rho and ras and promote breast carcinoma growth, migration, and invasion," Cancer Research, vol. 68, no. 19, pp. 7779-7787, 2008.

[20] R. M. Wolf, N. Draghi, X. Liang et al., "p190RhoGAP can act to inhibit PDGF-induced gliomas in mice: a putative tumor suppressor encoded on human Chromosome 19q13.3," Genes and Development, vol. 17, no. 4, pp. 476-487, 2003.

[21] A. Tikoo, S. Czekay, C. Viars et al., "p190-A, a human tumor suppressor gene, maps to the chromosomal region 19q13.3 that is reportedly deleted in some gliomas," Gene, vol. 257, no. 1, pp. 23-31, 2000.

[22] X. Long, D. Zhao, C. Wang et al., "Genetic polymorphisms in DNA repair genes XRCC4 and XRCC5 and aflatoxin B1-related hepatocellular carcinoma," Epidemiology, vol. 24, no. 5, pp. 671681, 2013.

[23] X. Long, J. Yao, Z. Zeng et al., "Polymorphisms in the coding region of X-ray repair complementing group 4 and aflatoxin B1related hepatocellular carcinoma," Hepatology, vol. 58, no. 1, pp. 171-181, 2013.

[24] A. C. Broders, "Squamous cell epithelioma of the skin: a study of 256 cases," Annals of Surgery, vol. 73, no. 2, pp. 141-160, 1921.
[25] A. F. Mavrogenis, C. N. Abati, C. Romagnoli, and P. Ruggieri, "Similar survival but better function for patients after limb salvage versus amputation for distal tibia osteosarcoma," Clinical Orthopaedics and Related Research, vol. 470, no. 6, pp. 17351748, 2012.

[26] H. Tamura, M. Fukada, A. Fujikawa, and M. Noda, "Protein tyrosine phosphatase receptor type $\mathrm{Z}$ is involved in hippocampus-dependent memory formation through dephosphorylation at Y1105 on p190 RhoGAP," Neuroscience Letters, vol. 399, no. 1-2, pp. 33-38, 2006.

[27] W. T. Arthur, L. A. Petch, and K. Burridge, "Integrin engagement suppresses RhoA activity via a c-Src-dependent mechanism," Current Biology, vol. 10, no. 12, pp. 719-722, 2000.

[28] K. Ludwig and S. J. Parsons, "The tumor suppressor, p190RhoGAP, differentially initiates apoptosis and confers docetaxel sensitivity to breast cancer cells," Genes and Cancer, vol. 2, no. 1, pp. 20-30, 2011.

[29] F. Mignone, C. Gissi, S. Liuni, and G. Pesole, "Untranslated regions of mRNAs," Genome Biology, vol. 3, no. 3, 2002.

[30] H. Notsuda, A. Sakurada, C. Endo et al., "p190A RhoGAP is involved in EGFR pathways and promotes proliferation, invasion and migration in lung adenocarcinoma cells," International Journal of Oncology, vol. 43, no. 5, pp. 1569-1577, 2013.

[31] I. Molina-Ortiz, R. A. Bartolomé, P. Hernández-Varas, G. P. Colo, and J. Teixidó, "Overexpression of E-cadherin on melanoma cells inhibits chemokine-promoted invasion involving p190RhoGAP/p120ctn-dependent inactivation of RhoA," Journal of Biological Chemistry, vol. 284, no. 22, pp. 15147-15157, 2009.

[32] G. Chakravarty, D. Roy, M. Gonzales, J. Gay, A. Contreras, and J. M. Rosen, "p190-B, a Rho-GTPase-activating protein, is differentially expressed in terminal end buds and breast cancer," Cell Growth and Differentiation, vol. 11, no. 7, pp. 343-354, 2000. 

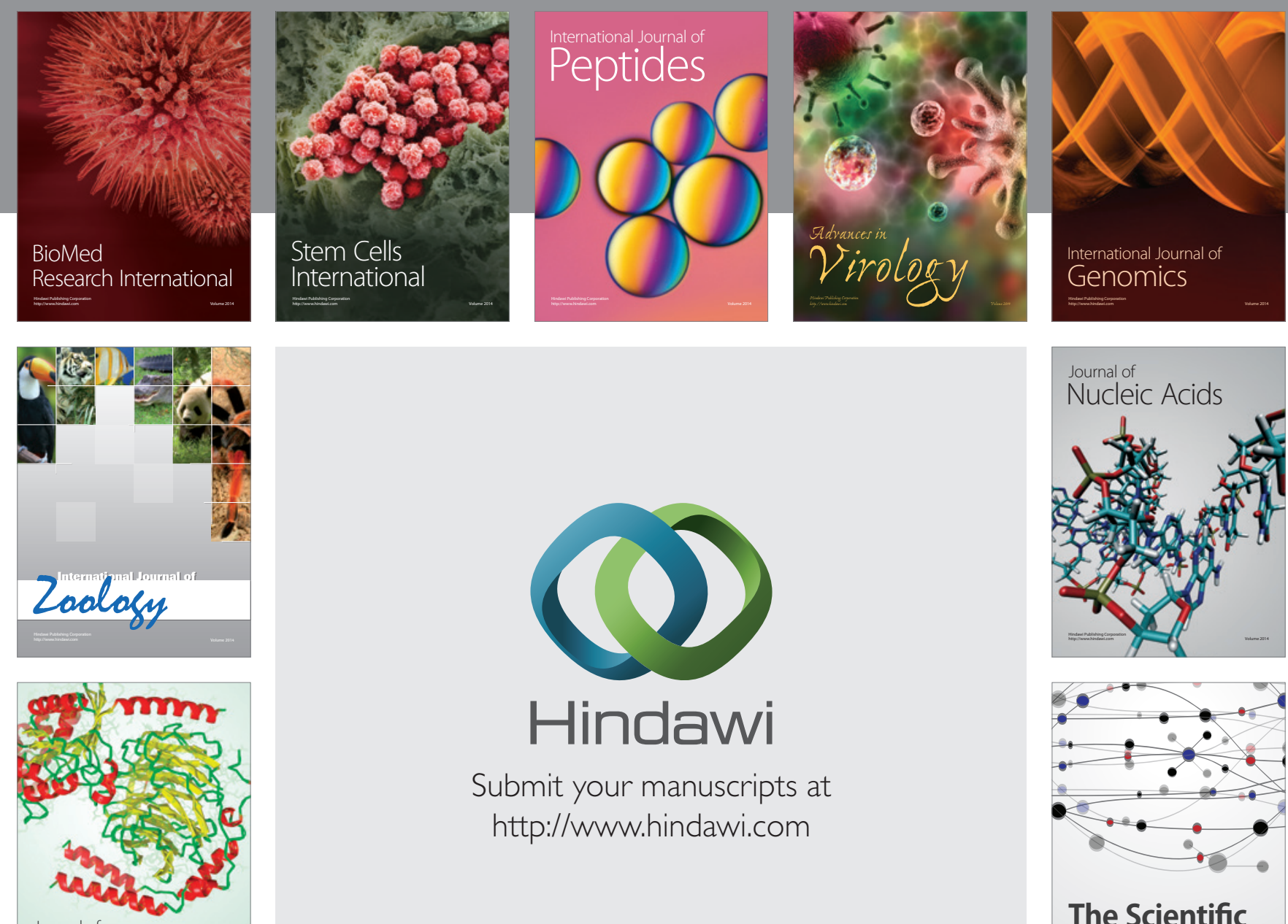

Submit your manuscripts at

http://www.hindawi.com

Journal of
Signal Transduction
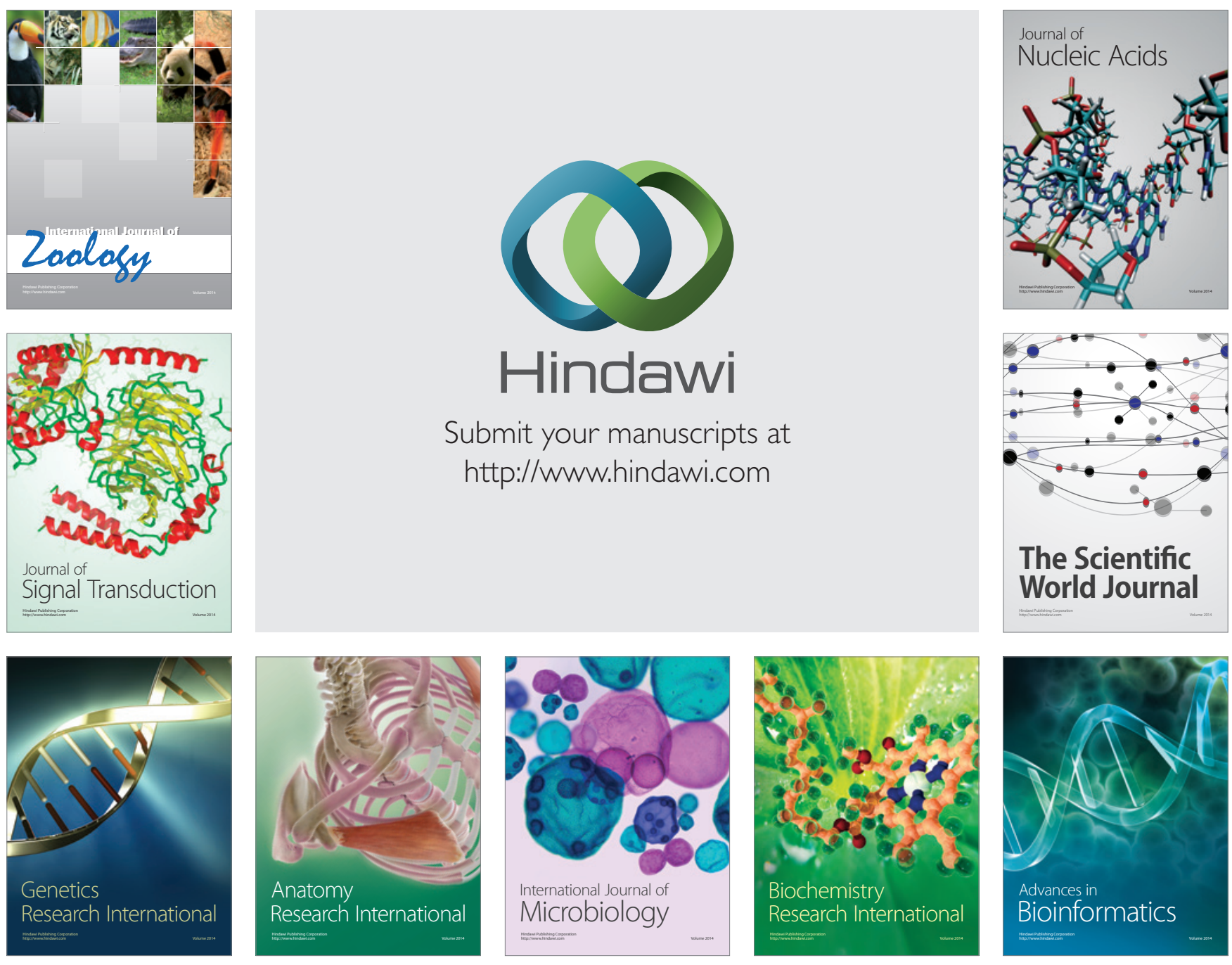

The Scientific World Journal
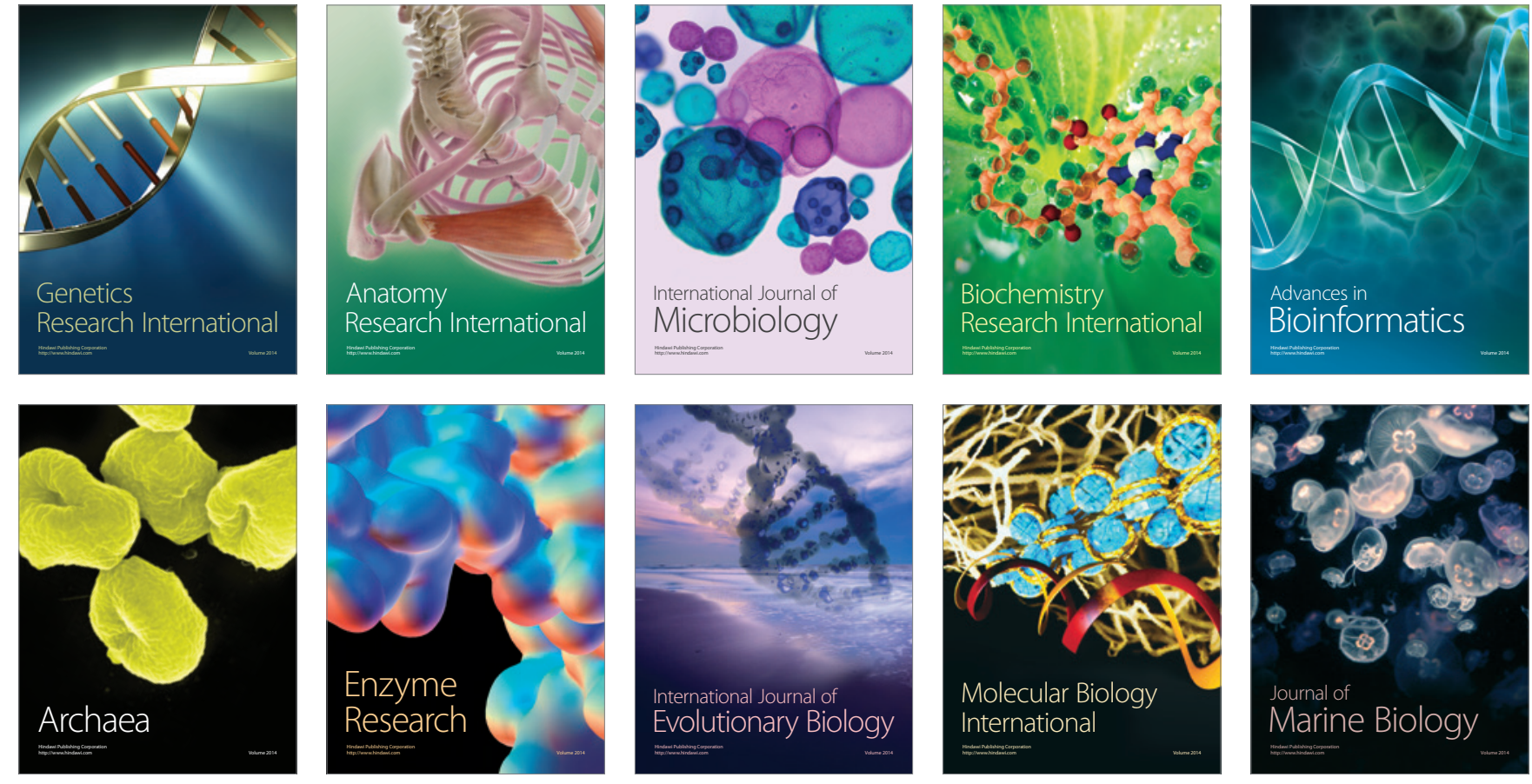